\title{
RECUPERAÇÃO DE PACIENTES COM LESÃO RENAL AGUDA DIALÍTICA E NÃO DIALÍTICA
}

\author{
Breno Guilherme Cardoso ${ }^{1}$, Tatiane Aguiar Carneiro ${ }^{2}$, Marcia Cristina da Silva Magro ${ }^{3}$
}

\begin{abstract}
RESUMO: O objetivo do estudo foi identificar a recuperação da função renal de pacientes com lesão renal aguda dialítica e não dialítica. Estudo observacional prospectivo e quantitativo, desenvolvido em unidade de terapia intensiva geral adulta de um hospital público do Distrito Federal, de janeiro a junho de 2015. Os dados foram coletados a partir de informações do prontuário eletrônico. Foram acompanhados 109 pacientes, desses 19 eram dialíticos, 10 (52,6\%) do sexo feminino, com idade média de $54 \pm 19$ anos. O grupo não dialítico constituiu-se por 90 pacientes predominantemente do sexo masculino 47 (52,2\%), idade média de $55 \pm 21$ anos. Entre os dois grupos, o dialítico apresentou como desfecho maior taxa de óbito $(73,7 \% ; \mathrm{p}=0,009)$ e maior valor de Simplified Acute Physiology Score III (83,0 $\pm 10,2 ; \mathrm{p}=0,0001)$. Apesar de o grupo dialítico acumular maior gravidade, o percentual de recuperação da função renal ocorreu em mais de $50 \%$ dos pacientes em ambos os grupos.
\end{abstract}

DESCRITORES: Lesão renal aguda; Unidade de terapia intensiva; Recuperação de função fisiológica; Enfermagem.

\section{RECOVERY OF PATIENTS WITH ACUTE KIDNEY INJURY REQUIRING DIALYSIS OR NOT}

\begin{abstract}
The research objective was to identify the recovery of the kidney function in patients with acute kidney injury requiring dialysis or not. Prospective and quantitative observational study, developed at an adult general intensive care unit of a public hospital in the Federal District, Brazil, between January and June 2015. The data were collected based on information from the electronic patient history. One hundred and nine patients were monitored, 19 of whom required dialysis and $10(52.6 \%)$ were female, with a mean age of $54 \pm 19$ years. The group that did not require dialysis consisted of 90 patients, predominantly male with $47(52.2 \%)$ and a mean age of $55 \pm 21$ years. Among the two groups, the outcomes in the dialysis group were a higher death rate $(73.7 \%$; $p=0.009)$ and higher Simplified Acute Physiology Score III (83.0 $10.2 ; p=0.0001)$. Although the dialysis group accumulates more severe patients, the recovery percentage of the kidney function corresponded to more than $50 \%$ of the patients in both groups.
\end{abstract}

DESCRIPTORS: Acute kidney injury; Intensive care units; Recovery of function; Nursing.

\section{RECUPERACIÓN DE PACIENTES CON LESIÓN RENAL AGUDA DIALÍTICA Y NO DIALÍTICA}

RESUMEN: El objetivo del estudio fue identificar la recuperación de la función renal de pacientes con lesión renal aguda dialítica y no dialítica. Estudio observacional prospectivo y cuantitativo, desarrollado en unidad de terapia intensiva general adulta de un hospital público del Distrito Federal, Brasil, de enero a junio del 2015. Los datos fueron recolectados a partir de informaciones del archivo electrónico. Fueron seguidos 109 pacientes, de los cuales 19 eran dialíticos, 10 (52,6\%) del sexo femenino, con promedio de $54 \pm 19$ años. El grupo no dialítico fue constituido por 90 pacientes predominantemente del sexo masculino 47 (52,2\%), promedio de edad $55 \pm 21$ años. Entre los dos grupos, el desfecho en el dialítico fue mayor tasa de óbito $(73,7 \% ; p=0,009)$ y mayor valor del Simplified Acute Physiology Score III $(83,0 \pm 10,2 ; p=0,0001)$. Aunque el grupo dialítico acumuló mayor gravedad, el porcentaje de recuperación de la función renal correspondió a más del $50 \%$ de los pacientes en ambos grupos.

DESCRIPTORES: Lesión renal aguda; Unidades de cuidados intensivos; Recuperación de la función; Enfermería.

${ }^{1}$ Enfermeiro. Universidade de Brasília. Brasília, DF, Brasil.

${ }^{2}$ Enfermeira. Especialista em Unidade de Terapia Intensiva. Secretaria Estadual de Saúde do Distrito Federal. Brasília, DF, Brasil. ${ }^{3}$ Enfermeira. Doutora em Enfermagem. Docente de Enfermagem da Universidade de Brasília. Brasília, DF, Brasil 


\section{- INTRODUÇÃO}

A lesão renal aguda (LRA) é definida como a redução aguda da função renal em horas ou dias, caracterizada tanto pela diminuição do ritmo de filtração glomerular e/ou do volume urinário, quanto pelos distúrbios no controle do equilíbrio hidroeletrolítico e ácido-base ${ }^{(1)}$. É uma patologia reversível e uma das complicações mais comuns do ambiente hospitalar, cuja incidência varia de acordo com a gravidade do paciente ${ }^{(2)}$.

A incidência da LRA em pacientes hospitalizados é de 5\%. Na unidade de terapia intensiva (UTI), além de esse percentual elevar-se para $17 \%$ a $35 \%$, acumula-se a necessidade de terapia dialítica para $49 \%$ a $70 \%$ dos pacientes. Sobretudo nos hospitais, a LRA torna-se importante complicação quando associada ao número e à gravidade das comorbidades apresentadas pelos pacientes ${ }^{(3-4)}$.

A incidência de LRA está aumentando especialmente entre os pacientes hospitalizados, em decorrência de procedimentos cirúrgicos, iatrogenias e sepse comuns ao cenário de UTI. Tais situações quando combinadas à idade e às comorbidades tornam os rins mais suscetíveis a insultos ${ }^{(5)}$.

Sendo assim, a identificação precoce da LRA pode representar um indicador para melhor direcionar a assistência e fornecer subsídios para a tomada de decisão. No âmbito gerencial e educacional, pode proporcionar o desenvolvimento de melhorias com vistas à construção de práticas resolutivas baseadas em evidências.

A classificação AKIN (Acute Kidney Injury Network) adotada neste estudo é uma ferramenta utilizada por profissionais de saúde, inclusive enfermeiros, para o estadiamento do grau de comprometimento da função renal. Essa classificação trouxe avanços importantes ${ }^{(6-7)}$, mas ainda depende dos valores de creatinina sérica e do volume urinário dos pacientes para identificação do grau de disfunção renal ${ }^{(8)}$.

A disfunção renal é definida pelo AKIN como o aumento no valor basal da creatinina sérica maior ou igual a 0,3mg/dL em um intervalo de pelo menos 48 horas. Segundo essa classificação, há três estágios de comprometimento da função renal. O estágio 1 corresponde ao risco de lesão renal, identificado pelo aumento absoluto da creatinina sérica $\geq 26,5 \mu \mathrm{mol} / \mathrm{L}(0,3 \mathrm{mg} / \mathrm{dL}$ ). $O$ estágio 2 (lesão renal) é caracterizado pelo aumento no valor basal da creatinina sérica maior do que 2 a 3 vezes; e no estágio 3 (falência renal), esse aumento é maior do que três vezes. Ainda no estágio 3 estão incluídos aqueles pacientes que necessitam de terapia de substituição renal(9).

Existem situações cujo agravo ao rim é tão intenso que há necessidade de instalação do tratamento dialítico (hemodiálise, diálise peritoneal ou hemofiltração) emergencial por haver risco iminente de complicações ao paciente. Entretanto, a melhor conduta é a prevenção da necessidade de diálise de urgência, pela prática da indicação precoce dessa terapia antes do surgimento do quadro de uremia e/ ou de complicações clínicas, metabólicas e eletrolíticas ${ }^{(1)}$.

Tal cenário exige do profissional de saúde competência para avaliação especializada, mas apesar dos avanços científicos, não há determinado sintoma ou sinal específico para identificar a LRA, o que limita e retarda o diagnóstico. Sabidamente, muitos pacientes recuperam a função renal, mas permanecem diálise-dependente, ou ainda com grave prejuízo da função renal por falta do estabelecimento de medidas precoces de prevenção(10).

A utilização de novas técnicas de diálise e dos avançados recursos disponíveis no cenário intensivo, apesar de proporcionarem o prolongamento da vida do paciente com LRA, não reduzem a mortalidade ${ }^{(11)}$.

Vários estudos em diferentes populações e ambientes hospitalares sinalizam que até mesmo formas "brandas" de LRA (isto é, associadas ao aumento na creatinina sérica de pelo menos 0,3 $\mathrm{mg} / \mathrm{dL}$ ) prenunciam significativa influência sobre a mortalidade ${ }^{(12-13)}$.

Nessa perspectiva, acredita-se que descrever os fatores relacionados à LRA e ao grau de recuperação da função renal de pacientes internados em uma UTI, permitirá identificar indicadores que poderão direcionar e individualizar as práticas assistenciais aos pacientes acometidos por essa patologia. 
Trata-se de um estudo observacional prospectivo, com abordagem quantitativa. O desenvolvimento do estudo ocorreu na UTI geral adulta de um hospital público do Distrito Federal, no período de janeiro a junho de 2015.

Para obtenção da estimativa do tamanho da amostra, foi utilizada a fórmula para o cálculo da proporção por meio do Software IBM Statistical Package for the Social Sciences (SPSS) Sample Power versão 3.0. A porcentagem de pacientes com LRA adotada foi $15 \%$, obtida a partir da incidência de lesão renal aguda em evidência científica ${ }^{(14)}$. Foi adotada a margem de erro na proporção de $7 \%$. O cálculo do tamanho amostral resultou em 100 pacientes. Considerando-se a perda de $8 \%$ dos dados, o tamanho da amostra calculado foi de 109 pacientes. Para os cálculos, o valor de alfa adotado foi de 95\%.

Foram incluídos no estudo pacientes com idade superior ou igual a 18 anos, que evoluíram com LRA dialítica e não dialítica segundo a classificação AKIN (Acute Kidney Injury Network) e excluídos os pacientes com diagnóstico médico de insuficiência renal crônica.

A estratégia de coleta de dados foi subsidiada pelo preenchimento de um questionário estruturado com dados de identificação do paciente, história clínica, evolução e parâmetros laboratoriais registrados no prontuário eletrônico do paciente.

Foi considerada como recuperação completa da função renal quando a proporção entre a creatinina sérica da alta da UTI/creatinina sérica de internação na UTI fosse menor ou igual a $20 \%$, e como recuperação parcial quando essa proporção fosse maior que $20 \%$, mas sem dependência de terapia de substituição renal ${ }^{(15)}$.

O banco de dados foi digitado no Programa SPSS versão 23. Os resultados foram expressos em média, desvio padrão, mediana (percentil 25\% e 75\%). A análise das variáveis categóricas foi realizada por meio do teste de Fisher ou Qui- quadrado. Foi aplicado o teste de Mann-Whitney para comparação entre variáveis categóricas. Foram considerados significativos p-values $<0,05$.

O projeto foi aprovado com o parecer no 942.648 do Comitê de Ética em Pesquisa da Fundação de Ensino e Pesquisa em Ciências da Saúde (FEPECS) da Secretaria Estadual de Saúde do Distrito Federal.

\section{RESULTADOS}

Dentre os 109 pacientes que evoluíram com LRA segundo os critérios da classificação AKIN, 19 necessitaram de tratamento dialítico durante a internação na UTI.

As características demográficas entre os grupos foram similares, particularmente idade, índice de massa corporal e raça. Ao contrário do grupo dialítico, o sexo masculino predominou no grupo não dialítico.

A hipertensão arterial sistêmica foi a comorbidade predominante, a sepse e a pneumonia destacaramse dentre os diagnósticos médicos presentes em ambos os grupos. O grupo dialítico apresentou como desfecho clínico a maior taxa de óbito. Nele, todos fizeram uso de ventilação mecânica com pressão positiva no final da expiração (PEEP), bem como utilizaram noradrenalina como droga vasoativa. Além disso, a maioria apresentou acidose. O índice Simplified Acute Physiology Score III (SAPS III) permaneceu elevado nos pacientes deste grupo $(p=0,0001)$.

Ao contrário do grupo dialítico, a maioria dos pacientes que não necessitou de diálise obteve alta como desfecho clínico. Em ambos os grupos, o emprego de noradrenalina, furosemida e de ventilação mecânica com PEEP foi expressivo, seguido de frequentes distúrbios metabólicos.

Tanto o uso de noradrenalina como de furosemida foram superiores no grupo dialítico e essa associação foi significativa $(p=0,003, p=0,02)$. A acidose e a alcalose ocorreram com maior frequência neste grupo de pacientes ( $p=0,02, p=0,0002)$, respectivamente.

A maioria dos pacientes do grupo não dialítico obteve alta da UTI $(p=0,009)$. Os resultados são 
apresentados nas Tabelas 1 e 2, abaixo.

Tabela 1 - Distribuição e análise univariada dos pacientes do grupo dialítico (GD) e não dialítico (GND) de acordo com as características demográficas e clínicas. Distrito Federal, Brasil, 2015

\begin{tabular}{|c|c|c|c|c|}
\hline Características & $\begin{array}{c}\text { GD } \\
\mathrm{n}=19(\%)\end{array}$ & $\begin{array}{c}\text { GND } \\
\mathrm{n}=90(\%)\end{array}$ & $\begin{array}{c}\text { Todos } \\
\mathrm{n}=109(\%) \\
\end{array}$ & Valor $p$ \\
\hline Idade (anos) & $54 \pm 19$ & $55 \pm 21$ & $54 \pm 21$ & $0,8^{*}$ \\
\hline Sexo masculino & $9(47,4)$ & $47(52,2)$ & $56(51,4)$ & $0,9^{*}$ \\
\hline Índice de massa corporal (kg/m2) & $25,7 \pm 7,3$ & $25,3 \pm 5,3$ & $25,3 \pm 5,7$ & $0,8^{*}$ \\
\hline \multicolumn{5}{|l|}{ Raça } \\
\hline Branca & $2(10,5)$ & $16(17,8)$ & $18(16,5)$ & $0,3+$ \\
\hline Parda & $15(79)$ & $49(54,4)$ & $64(58,7)$ & $0,08+$ \\
\hline Negra & $2(10,5)$ & $25(27,8)$ & $27(24,8)$ & $0,09+$ \\
\hline \multicolumn{5}{|l|}{ Comorbidades } \\
\hline Hipertensão arterial & $7(36,8)$ & $35(38,9)$ & $42(38,5)$ & $0,9+$ \\
\hline Diabetes Mellitus & $4(21,1)$ & $21(23,3)$ & $25(22,9)$ & $0,5+$ \\
\hline Doença pulmonar obstrutiva crônica & $5(26,3)$ & $16(17,8)$ & $21(19,3)$ & $0,3+$ \\
\hline Cardiopatia & $3(15,8)$ & $16(17,8)$ & $19(17,4)$ & $0,6+$ \\
\hline Tireoideopatia & $0(0,0)$ & $3(3,3)$ & $3(2,8)$ & $0,6+$ \\
\hline Cirrose & $0(0,0)$ & $1(1,1)$ & $1(0,9)$ & $0,8+$ \\
\hline Etilista & $2(10,5)$ & $11(12,2)$ & $13(11,9)$ & $0,6+$ \\
\hline Tabagista & $4(21,1)$ & $20(22,2)$ & $24(22)$ & $0,6+$ \\
\hline Acidente vascular encefálico & $1(5,3)$ & $9(10)$ & $10(9,2)$ & $0,4+$ \\
\hline \multicolumn{5}{|l|}{ Diagnóstico } \\
\hline Sepse & $10(52,6)$ & $40(44,4)$ & $50(45,9)$ & $0,7+$ \\
\hline Insuficiência respiratória aguda & $3(15,8)$ & $23(25,6)$ & $26(23,9)$ & $0,4+$ \\
\hline Edema agudo de pulmão & $0(0,0)$ & $5(5,6)$ & $5(4,6)$ & $0,8+$ \\
\hline Pneumonia & $8(42,1)$ & $38(42,2)$ & $46(42,2)$ & $0,3+$ \\
\hline POI de Laparotomia exploradora & $3(15,8)$ & $9(10)$ & $12(11)$ & $0,4+$ \\
\hline Acidente vascular encefálico & $1(5,3)$ & $10(11,1)$ & $11(10,1)$ & $0,4 \neq$ \\
\hline
\end{tabular}

Legenda:

*Teste de Mann Whitney

†Teste Qui-quadrado

¥ Teste exato de Fisher

POI=pós-operatório imediato

Tabela 2 - Análise univariada dos pacientes do grupo dialítico (GD) e não dialítico (GND) de acordo com as variáveis clínicas e desfecho. Distrito Federal, Brasil, 2015 (continua)

\begin{tabular}{lcccc} 
Características & $\begin{array}{c}\text { GD } \\
\mathbf{n = 1 9}\end{array}$ & $\begin{array}{c}\text { GND } \\
\mathbf{n = 9 0}\end{array}$ & $\begin{array}{c}\text { Todos } \\
\mathbf{n = 1 0 9}\end{array}$ & Valor $\mathbf{p}$ \\
\hline Uso de drogas & $19(100,0)$ & $61(67,8)$ & $80(73,4)$ & \\
\hline Noradrenalina & $19(100,0)$ & $56(62,2)$ & $75(68,8)$ & $0,003+$ \\
\hline Nipride & $1(5,3)$ & $17(18,9)$ & $18(16,5)$ & $0,1+$ \\
\hline Furosemida & $18(94,7)$ & $63(70,0)$ & $81(74,3)$ & $0,02 \ddagger$ \\
\hline Pressão arterial média alterada $(<70$ ou $>100)$ & $19(100,0)$ & $89(98,9)$ & $108(99,1)$ & $0,8 \neq$ \\
\hline Apresentou alcalose & $5(26,3)$ & $64(71,1)$ & $69(63,3)$ & $0,0002+$ \\
\hline Apresentou acidose & $18(94,7)$ & $62(68,9)$ & $80(73,4)$ & $0,02 \ddagger$ \\
\hline Índice SAPS & $83,0 \pm 10,2$ & $68,3 \pm 12,2$ & $70,7 \pm 13,1$ & $0,0001^{*}$ \\
\hline Paciente cirúrgico & $8(42,1)$ & $24(26,7)$ & $32(29,4)$ & $0,3+$ \\
\hline \hline
\end{tabular}




\begin{tabular}{lcccc}
\hline Uso ventilação mecânica & $19(100,0)$ & $78(86,7)$ & $97(89,0)$ & $0,06+$ \\
\cline { 1 - 4 } \cline { 1 - 3 } Tempo de ventilação mecânica (dias) & $22,4 \pm 14,8$ & $32,4 \pm 34,2$ & $30,5 \pm 31,5$ & \multirow{2}{*}{$0,4^{*}$} \\
\cline { 2 - 4 } & $16(12-39)$ & $22(12-41)$ & $20(12-39)$ & \\
\hline PEEP & $12,3 \pm 1,6$ & $12,6 \pm 11,7$ & $12,6 \pm 10,5$ & $0,03^{*}$ \\
\hline Desfecho & & & & \\
\hline Alta & $5(26,3)$ & $55(61,1)$ & $60(55,1)$ & $0,009+$ \\
\hline Óbito & $14(73,7)$ & $33(36,7)$ & $47(43,1)$ & \\
\hline Disfunção renal & $19(100,0)$ & $86(95,6)$ & $105(96,3)$ & $0,5 \ddagger$
\end{tabular}

Legenda:

$\mathrm{PEEP}=$ pressão positiva no final da expiração

SAPS III = Simplified Acute Physiology Score III

₹ Teste exato de Fisher

* Teste de Mann Whitney

tTeste Qui-quadrado

O comprometimento da função renal nos grupos dialítico e não dialítico está evidenciado na Tabela 3, entretanto destaca-se que a gravidade do acometimento renal foi superior no grupo dialítico, pois 17 (89,5\%) evoluíram com falência renal pelo critério volume urinário e 12 (63,2\%) pelo critério creatinina, ressaltando que essas duas variáveis subsidiam a avaliação da função renal pela classificação AKIN.

Tabela 3 - Estadiamento da função renal segundo a classificação AKIN dos pacientes do grupo dialítico (GD) e do grupo não dialítico (GND) durante a internação na Unidade de Terapia Intensiva. Distrito Federal, Brasil, 2015

\begin{tabular}{|c|c|c|c|c|c|}
\hline GD $(n=19)$ & & & GND $(n=90)$ & & \\
\hline Estágio & $\begin{array}{c}\text { Critério } \\
\text { creatinina }\end{array}$ & $\begin{array}{c}\text { Critério volume } \\
\text { urinário }\end{array}$ & Estágio & $\begin{array}{c}\text { Critério } \\
\text { creatinina }\end{array}$ & $\begin{array}{c}\text { Critério volume } \\
\text { urinário }\end{array}$ \\
\hline 1 (Risco) & $2(10,5)$ & $0(0,0)$ & 1 (Risco) & $29(32,2)$ & $13(14,4)$ \\
\hline 2 (Lesão) & $2(10,5)$ & $2(10,5)$ & 2 (Lesão) & $7(7,8)$ & $39(43,3)$ \\
\hline 3 (Falência) & $12(63,2)$ & $17(89,5)$ & 3 (Falência) & $10(11,1)$ & $30(33,3)$ \\
\hline
\end{tabular}

Entre os grupos dialítico e não dialítico, a recuperação da função renal foi mais expressiva no primeiro, como pode ser verificado na Tabela 4. Vale ressaltar que a recuperação da função renal foi avaliada em 37 dos 109 pacientes, por motivo de óbito ou alta hospitalar dos demais pacientes.

Tabela 4 - Recuperação da função renal dos pacientes dialíticos (GD) e não dialíticos (GND), distribuída de acordo com os meses de acompanhamento. Distrito Federal, Brasil, 2015

\begin{tabular}{lcc} 
Recuperação da função renal & GD & GND \\
\cline { 2 - 3 } & $\mathbf{( n = 4 )}$ & $\mathbf{( n = 3 3 )}$ \\
\hline A partir do $1^{\circ}$ mês & $1(25,0)$ & $14(42,4)$ \\
\hline A partir do $2^{\circ}$ mês & $2(50,0)$ & $1(3,0)$ \\
\hline A partir do $3^{\circ}$ mês & $0(0,0)$ & $2(6,1)$ \\
\hline Total de pacientes que recuperaram a função renal & $3(75,0)$ & $17(51,5)$
\end{tabular}

*86 pacientes não possuíam registro da creatinina nesses períodos. 


\section{- DISCUSSÃO}

A LRA é previsível e pode ser evitável se fatores de risco são identificados precocemente no contexto clínico. Seguramente, quando a detecção da LRA não diagnosticada é oportuna, também pode levar a uma melhor gestão da doença ${ }^{(16)}$.

Evidência científica com 477 pacientes com diagnóstico de LRA por necrose tubular aguda, internados em um hospital de ensino, identificou o sexo masculino e a idade média de $65 \pm 16,2$ anos como condições comuns em pacientes que evoluem com LRA ${ }^{(4)}$. Os achados do estudo ora apresentado mostram características semelhantes no grupo não dialítico. Por outro lado, em outro estudo, com 117 pacientes admitidos em uma UTI de hospital de ensino, o percentual do sexo feminino foi mais expressivo, assim como evidenciado no grupo dialítico deste estudo.

Dessa forma, identifica-se a falta de consenso até os dias atuais em se afirmar o sexo de maior incidência da LRA entre os estudos, considerando que geralmente essa variação ocorre em função da definição de LRA assumida nos estudos e das características da população estudada, haja vista que atualmente possuímos três classificações distintas para identificação e avaliação da função renal, a saber RIFLE, do acrônimo risk, injury, failure, loss, end-stage, AKIN (acute kidney injury network) e KDIGO (Kidney Disease Improving Global Outcomes) ${ }^{(17)}$.

A senescência ou envelhecimento fisiológico normal retrata as alterações renais relacionadas com a idade. As mudanças estruturais microanatômicas do rim com a idade mais avançada incluem a redução do número de glomérulos funcionais em decorrência do aumento da prevalência da nefroesclerose (arteriosclerose, esclerose glomerular, atrofia tubular e a fibrose intersticial), e, em certa medida, pela hipertrofia compensatória de néfrons remanescentes ${ }^{(18)}$. Nesse contexto, a idade avançada representa um fator predisponente à ocorrência da LRA. Característica similar foi identificada nos pacientes deste estudo.

Sabidamente, alguns fatores de risco e/ou comorbidades podem modificar a probabilidade de recuperação da LRA e resultar em piora do quadro do paciente. A idade avançada ( $>60$ anos), a presença de comorbidades e o início tardio da terapia de substituição renal destacam-se entre eles ${ }^{(19)}$. Além disso, creatinina sérica basal maior que $1,2 \mathrm{mg} / \mathrm{dL}$, o uso prévio de anti-inflamatórios não esteroidais, presença de choque séptico e a hipertensão arterial sistêmica representam também outros fatores relacionados à ocorrência de LRA ${ }^{(10)}$. Alguns desses fatores também foram encontrados neste estudo.

A mortalidade elevada de pacientes com LRA internados na UTI é uma realidade no panorama atual. Há uma variedade de fatores que predispõe à LRA, acarretando sofrimento ao paciente, internação hospitalar prolongada, necessidade de terapia de substituição renal, e até mesmo a morte ${ }^{(20)}$.

O desenvolvimento de complicações como infecções, sepse, hemorragias, cirurgias e necessidade de diálise durante a internação em UTI podem aumentar a gravidade do paciente e da LRA. Esses fatores, combinados ou isolados, também podem predispor à maior mortalidade ${ }^{(10)}$. Em nosso estudo, esse desfecho foi sinalizado pela elevação do SAPS III, índice preditivo de mortalidade composto de 20 variáveis, representadas por escore fisiológico agudo e avaliação do estado prévio de pacientes admitidos em unidades de terapia intensiva ${ }^{(21)}$.

Estudo mostrou que de um total de 152 pacientes estratificados em dialíticos e não dialíticos, verificou-se que todos os dialíticos e $56,4 \%$ dos não dialíticos utilizaram ventilação mecânica invasiva e $52,6 \%$ dos dialíticos e $25,6 \%$ dos não dialíticos evoluíram com sepse ${ }^{(22)}$. Ao compararmos os dados deste estudo com outros encontrados ${ }^{(22-23)}$, constatou-se similaridades com relação as características clínicas e, principalmente, nos desfechos. No entanto, neste estudo, esses fatores de risco, por mais que tenham sido evidentes em ambos os grupos, sempre se sobressaíram no grupo dialítico, com mortalidade mais elevada.

Evidência científica mostrou que os principais fatores de risco para LRA também podem estar relacionados às doenças cardiológicas (hipertensão arterial sistêmica, insuficiência cardíaca congestiva, hipovolemia), choque séptico, síndrome da resposta inflamatória sistêmica (SIRS), assim como a utilização de furosemida ${ }^{(22)}$. Achado também encontrado neste estudo. 
Um estudo ${ }^{(22)}$ reforça os resultados da nossa pesquisa ao verificar que o paciente dialítico e o não dialítico possuem praticamente os mesmos fatores de riscos, e que na realidade estes comportam-se como indicadores para melhor gestão do cuidado. Entretanto, os pacientes submetidos à terapia renal substitutiva, por apresentarem um quadro clínico potencialmente mais grave, são mais suscetíveis a procedimentos invasivos e a eventos adversos. No estudo ora apresentado, o grupo que realizou tratamento dialítico apresentou maior taxa de óbito, mas acredita-se que tal fato não pode ser atribuído isoladamente ao tratamento dialítico.

Atualmente, é consenso universal o emprego de classificações multidimensionais como a AKIN, adotada neste estudo, para identificar e estadiar a LRA, porém ainda não existe uma definição clara e objetiva da recuperação renal após o evento de LRA. Isso pode retardar as ações assistenciais e aumentar a gravidade do paciente ${ }^{(20)}$.

Neste estudo, o grupo dialítico desenvolveu acidose metabólica. Evidência científica ratifica a acidose metabólica grave como fator de risco isolado para mortalidade em pacientes dialíticos com LRA, sendo até mesmo necessário intervenção precoce para correção visando melhorar as chances de sobrevida ${ }^{(24)}$.

Em estudo(23) a LRA segundo AKIN, critério creatinina sérica, mostrou que a maioria (69,3\%) dos pacientes encontravam-se com LRA no estágio 3 (falência renal). Da mesma forma, neste estudo, a maioria dos pacientes dialíticos foi classificada no estágio de falência, não só pelo critério creatinina sérica, mas também pelo critério volume urinário, enquanto no grupo não dialítico o estágio 2 (lesão renal), de menor gravidade, acometeu a maioria dos pacientes.

Em relação às limitações e implicações para pesquisas futuras, a qualidade e a dificuldade de acesso aos registros dos prontuários eletrônicos representaram as limitações deste estudo. Por outro lado, os achados deste estudo possibilitarão melhor direcionamento do cuidado e facilitarão a implementação de práticas resolutivas no processo saúde e doença, baseada em evidências.

\section{CONCLUSÃO}

A LRA é complicação frequente no cenário da UTI, geralmente relacionada a outras patologias e medicamentos. Observou-se o quantitativo expressivo de pacientes, de ambos os grupos (dialítico e não dialítico) que evoluíram com lesão ou falência renal de acordo com a classificação AKIN.

Ainda nos dias atuais o uso de drogas vasoativas como a noradrenalina, ventilação mecânica, distúrbios metabólicos podem predispor a ocorrência da LRA.

Apesar de o grupo dialítico acumular maior gravidade, o percentual de recuperação da função renal ocorreu em mais de $50 \%$ em ambos os grupos.

\section{REFERÊNCIAS}

1. Sociedade Brasileira de Nefrologia (SBN). Diretrizes da Sociedade Brasileira de Nefrologia: Insuficiência renal aguda. Comitê de Insuficiência Renal Aguda da Sociedade Brasileira de Nefrologia. JBN. [Internet] 2007 [acesso em 05 dez 2015]. Disponível: http://sbn.org.br/app/uploads/Diretrizes_Insuficiencia_Renal_Aguda.pdf.

2. Santos ES, Marinho CMS. Principais causas de insuficiência renal aguda em unidades de terapia intensiva: intervenção de enfermagem. Rev. Enf. Ref. [Internet] 2013;III(9) [acesso em 13 set 2016]. Disponível: http://dx.doi. org/10.12707/RIII1272.

3. Bernardina LD, Diccini S, Belasco AGS, Bittencourt ARC, Barbosa DA. Evolução clínica de pacientes com insuficiência renal aguda em unidade de terapia intensiva. Acta paul. enferm. [Internet] 2008;21(n.esp) [acesso em 12 set 2016]. Disponível: http://dx.doi.org/10.1590/S0103-21002008000500007.

4. Bucuvic EM, Ponce D, Balbi AL. Fatores de risco para mortalidade na lesão renal aguda. Rev. Assoc. Med. Bras. [Internet] 2011;57(2) [acesso em 12 set 2016]. Disponível: http://dx.doi.org/10.1590/S0104-42302011000200012. 
5. Hoste EA, Bagshaw SM, Bellomo R, Cely CM, Colman R, Cruz DN, et al. Epidemiology of acute kidney injury in critically ill patients: the multinational AKI-EPI study. Intensive Care Med. [Internet] 2015;41(8) [acesso em 13 set 2016]. Disponível: http://dx.doi.org/10.1007/s00134-015-3934-7.

6. Kellum JA, Lameire N, KDIGO AKI Guideline Work Group. Diagnosis, evaluation, and management of acute kidney injury: a KDIGO summary (Part 1). Crit Care. [Internet] 2013;17(1) [acesso em 13 set 2016]. Disponível: http://dx.doi.org/10.1186/cc11454.

7. Kellum JA, Bellomo R, Ronco C. Kidney attack. JAMA. [Internet] 2012;307(21) [acesso em 13 set 2016 ]. Disponível: http://dx.doi.org/10.1001/jama.2012.4315.

8. Levi TM, de Souza SP, de Magalhães JG, de Carvalho MS, Cunha ALB, Dantas JGAO, et al. Comparação dos critérios RIFLE, AKIN e KDIGO quanto a capacidade de predição de mortalidade em pacientes graves. Rev. bras. ter. intensiva. [Internet] 2013;25(4) [acesso em 12 set 2016]. Disponível: http://dx.doi.org/10.5935/0103507X.20130050.

9. Mehta RL, Kellum JA, Shah SV, Molitoris BA, Ronco C, Warnock DG, et al. Acute Kidney Injury Network (AKIN): report of an initiative to improve outcomes in acute kidney injury. Crit Care. [Internet] 2007;11(2) [acesso em 01 dez 2016]. Disponível: http://dx.doi.org/10.1186/cc5713.

10. Macedo E, Malhotra R, Claure-Del Granado R, Fedullo P, Mehta RL. Defining urine output criterion for acute kidney injury in critically ill patients. Nephrol Dial Transplant. [Internet] 2011;26(2) [acesso em 13 set 2016]. Disponível: http://dx.doi.org/10.1093/ndt/gfq332.

11. Ponce D, Zorzenon CPF, dos Santos NY, Teixeira UA, Balbi AL. Injúria renal aguda em unidade de terapia intensiva: estudo prospectivo sobre a incidência, fatores de risco e mortalidade. Rev. bras. ter. intensiva. [Internet] 2011;23(3) [acesso em 12 set 2016]. Disponível: http://dx.doi.org/10.1590/S0103-507X2011000300010.

12. Hoste EA, Kellum JA. RIFLE criteria provide robust assessment of kidney dysfunction and correlate with hospital mortality. Crit Care Med. [Internet] 2006;34(7) [acesso em 13 set 2016]. Disponível: http://www.ncbi.nlm. nih.gov/pubmed/16801870.

13. Uchino S, Bellomo R, Goldsmith D, Bates S, Ronco C. An assessment of the RIFLE criteria for acute renal failure in hospitalized patients. Crit Care Med. [Internet] 2006;34(7) [acesso em 13 set 2016]. Disponível: https://www. ncbi.nlm.nih.gov/pubmed/16715038.

14. Doyle JF, Forni LG. Long-term follow-up of acute kidney injury. Crit Care Clin. [Internet] 2015;31(4) [acesso em 12 set 2016]. Disponível: http://dx.doi.org/10.1016/j.ccc.2015.06.017.

15. Macedo E, Bouchard J, Mehta RL. Renal recovery following acute kidney injury. Curr Opin Crit Care. [Internet] 2008;14(6) [acesso em 13 set 2016]. Disponível: http://dx.doi.org/10.1097/MCC.0b013e328317ee6e.

16. Kate RJ, Perez RM, Mazumdar D, Pasupathy KS, Nilakantan V. Prediction and detection models for acute kidney injury in hospitalized older adults. BMC Med Inform Decis Mak. [Internet] 2016;(16) [acesso em 13 set 2016]. Disponível: http://dx.doi.org/10.1186/s12911-016-0277-4.

17. Koza Y. Acute kidney injury: current concepts and new insights. J Inj Violence Res. [Internet] 2016;8(1) [acesso em 13 set 2016]. Disponível: http://dx.doi.org/10.5249/jivr.v8i1.610.

18. Denic A, Glassock RJ, Rule AD. Structural and Functional Changes With the Aging Kidney. Adv Chronic Kidney Dis. [Internet] 2016;23(1) [acesso em 13 set 2016]. Disponível: http://dx.doi.org/10.1053/j.ackd.2015.08.004.

19. Pôncio L, Balbi AL, da Rocha EP, Dias DB, Ponce D. Evolução em longo prazo após episódio de lesão renal aguda: revisão narrativa. J. Bras. Nefrol. [Internet] 2015;37(1) [acesso em 9 jan 2016]. Disponível: http://dx.doi. org/10.5935/0101-2800.20150016.

20. Santos JCO, Mendonça MAO. Fatores predisponentes para lesão renal aguda em pacientes em estado crítico: revisão integrativa. Rev. Soc. Bras. Clin. Med. [Internet] 2015;13(1) [acesso em 9 jan 2016]. Disponível: http://files. bvs.br/upload/S/1679-1010/2015/v13n1/a4780.pdf.

21. Silva Junior JM, Malbouisson LMS, Nuevo HL, Barbosa LGT, Marubayashi LY, Teixeira IC, et al. Aplicabilidade do escore fisiológico agudo simplificado (SAPS 3) em hospitais brasileiros. Rev. Bras. Anestesiol. [Internet] 
2010;60(1) [acesso em $01 \mathrm{dez} 2016]$. Disponível: http://dx.doi.org/10.1590/S0034-70942010000100003.

22. Peres LAB, Wandeur V, Matsuo T. Preditores de injúria renal aguda e de mortalidade em uma Unidade de Terapia Intensiva. J. Bras. Nefrol. [Internet] 2015;37(1) [acesso em 9 jan 2016]. Disponível: http://dx.doi. org/10.5935/0101-2800.20150007.

23. de Souza SP, Matos RS, Barros LL, Rocha PN. Associação inversa entre creatinina sérica e mortalidade na lesão renal aguda. J. Bras. Nefrol. [Internet] 2014;36(4) [acesso em 12 set 2016]. Disponível: http://dx.doi. org/10.5935/0101-2800.20140067.

24. Annigeri RA, Nandeesh V, Karuniya R, Rajalakshmi S, Venkataraman R, Ramakrishnan N. Impact of dialysis practice patterns on outcomes in acute kidney injury in Intensive Care Unit. Indian J Crit Care Med. [Internet] 2016;20(1) [acesso em 29 mai 2016]. Disponível: http://dx.doi.org/10.4103/0972-5229.173682. 